\title{
AUTHORS:
}

Please follow this template as closely as possible when formatting your article. It contains specific "Styles" in Microsoft Word. If you have any questions, contact the MRS Bulletin editorial office: Lori Wilson, technical editor, lwilson@mrs.org.

\section{Analytical Electron Tomography}

Rowan K. Leary and Paul A. Midgley*

This short review highlights recent advances in analytical electron tomography (AET), the 3D extension of conventional nano-analytical techniques, in which electron energy-loss and X-ray spectroscopy and electron diffraction are combined with tomographic acquisition and reconstruction. We show key examples from the literature illustrating how new 3D information, gleaned from AET, provides insights into not just morphology and composition, but also the electronic, chemical and optical properties of materials at the nanoscale. We describe how the 'multi-dimensional' nature of AET leads to 'big data' sets, how these can be analyzed optimally and look forward to how AET may develop further.

Keywords: scanning transmission electron microscopy (STEM), nanoscale, electron energy loss spectroscopy (EELS), x-ray tomography, crystallographic structure.

\section{Introduction}

Over the past 15 years or so, electron tomography using the (scanning) transmission electron microscope ((S)TEM) has become a technique used routinely to determine the $3 \mathrm{D}$ nanoscale morphology of a variety of materials. ${ }^{1-4}$ As described elsewhere in this issue of MRS Bulletin, a tilt series of images are acquired which, if considered as a series of projections, can be 'back-projected' to reconstruct the 3D object of interest. STEM high-angle annular dark-field 
(HAADF) imaging, which has become the de facto standard for electron tomography of strongly scattering crystalline specimens in the physical sciences, provides some compositional contrast through the atomic number dependence of the high-angle scattered electrons, but often a more direct determination of composition is needed through electron energy-loss spectroscopy (EELS) and/or energy-dispersive X-ray spectroscopy (EDXS). Combining spectroscopy and tomography has proven to be a valuable method to determine not just $3 \mathrm{D}$ composition, but also chemistry (e.g. local valency), electronic properties and optical properties. More recently the ability to undertake 3D nano-crystallography has added to the ensemble of 3D nanoscale imaging techniques now available which, together, we will call here 'analytical electron tomography' (AET), see Figure 1.

We note that other advanced techniques available in electron microscopy can be coupled to tomography, electron holography in particular. ${ }^{5}$ Indeed the number of different imaging modes used in electron tomography has seen five-fold growth over the last 15 years. While some of these could also be considered under the umbrella of 'AET', we focus the present review on the aforementioned due to: (i) EELS, EDXS and diffraction being the most widespread techniques in analytical electron microscopy in general; (ii) EELS and EDXS being the most widely implemented forms of AET to date; and (iii) crystallographic tomography being an area pursued in our research group in which we anticipate notable future development.

\section{EFTEM and EELS Tomography}

An electron spectrometer, placed either in-column or post-column, enables the electron beam, having traversed through the sample, to be dispersed in energy and an energy-loss spectrum formed. This spectrum can be collected pixel by pixel over a region of interest, a technique known as STEM spectrum-imaging (described later), or using 'energy-filtered TEM' (EFTEM) an energy window 
may be placed over a characteristic energy-loss feature and an image formed using only those electrons that lie within that energy window.

For EFTEM using core-loss ionization edges, the background signal under the edge can be estimated from additional EFTEM images (normally two, recorded before the loss), and then subtracted from the core-loss EFTEM image to yield an elemental map. By acquiring a tilt series of such elemental maps, and using those as input for tomographic reconstruction, a 3D element-sensitive tomogram can be reconstructed. In Figure 2(a), for example, a 3D iron elemental map shows clearly an iron-based catalyst nanoparticle at the top of a multi-wall carbon nanotube (CNT). Knowing the 3D morphology of each chemical constituent in the ironfilled CNTs is one of the key factors for understanding the growth mechanism and potential applications. ${ }^{6}$

Whilst EFTEM maps and the associated tilt series are relatively straightforward to acquire (indeed EFTEM-based AET was first demonstrated more than 15 years $\operatorname{ago}^{7,8}$ ), the spectral information contained within the EFTEM series is limited energy windows are often ca. $20 \mathrm{eV}$ in size and so subtle spectroscopic information (e.g. edge fine structure) may be lost. This limited spectral information has led to an increasing adoption of STEM-EELS spectrum-imaging, in which the complete energy-loss spectrum is acquired at every pixel. By recording a tilt series of such spectrum-images, 3D tomograms may be reconstructed from any energy-loss channel recorded in the spectrum. Using coreloss ionization edges, the elemental signal can be extracted from each spectrum pixel-by-pixel, and elemental maps at each tilt can then be used as inputs into tomographic reconstruction. If, instead, every channel in the EELS spectrum is reconstructed tomographically, it is possible to obtain 'voxel spectra', which can be analyzed subsequently.

The fine structure seen at core-loss ionization edges may encode information on the electronic and bonding properties of the sample. As an example, shown in 
Figure 2(b), by fitting model ionization edges to experimental spectra, Jarausch et al. mapped in $3 \mathrm{D}$ the bonding states of silicon in a semiconductor device; the silicon is identified as either in its elemental form, as an oxide or as part of a metal silicide or silicon nitride. ${ }^{9}$

Electronic information is encoded also in the low-loss region of the EELS spectrum. The bulk, or volume, plasmon energy depends on the local electron density and plasmon EFTEM images, using narrow energy windows, ca. 1-2 eV in width, may be used as input for tomographic reconstructions. Figure 2 (c) shows an early example in which islands of silicon can be distinguished from the silicon oxide matrix by the shift in the plasmon energy. ${ }^{10}$

In favorable cases it may be possible to map directly in 3D the valency of certain elements. Materials with empty $3 \mathrm{~d}$ and $4 \mathrm{f}$ shells are amenable to this because of very pronounced and intense EELS fine structure that can be used as a fingerprint to determine the valency. The example in Figure 2(d) shows a ceria nanoparticle in which the particle surface is shown to be predominantly $\mathrm{Ce}^{3+}$ in character and the core $\mathrm{Ce}^{4+} \cdot 11$

In the EFTEM and EELS cases cited above, the energy-loss signal increases with the projected sample thickness and thus is suitable for reconstruction. However, once the sample thickness extends beyond some characteristic inelastic mean free path (typically $100 \mathrm{~nm}$ or so ${ }^{12}$ ) the elemental signal may actually fall, as the influence of plural (multiple inelastic) scattering becomes significant. This creates a problem for reconstruction. Most tomography reconstruction relies still on a back-projection approach in which the image signal used is in some sense a 'projection' and is assumed to vary monotonically with some physical quantity (e.g. thickness). For thick specimens, the core-loss signal no longer satisfies the projection requirement. Methods to remove the effects of plural scattering are available, but their implementation in EELS and EFTEM tomography are very limited to date. With the introduction of so-called 'dual EELS' technology, in 
which the low loss and core loss spectra are acquired 'simultaneously', this plural scattering correction may become more reliable and used routinely.

\section{EDXS Tomography}

In many ways, STEM-EDX provides a much simpler method with which to form a compositional map. The characteristic EDXS peaks lie on a relatively low background, and there is little of the plural scattering problems that plagues EELS and EFTEM of thick specimens. Until recently though, the major problem has primarily been the detector geometry. Most conventional EDXS detectors used in (S)TEM subtended solid angles of 0.1-0.3 sr, and were positioned on one side of the electron microscope column. Thus, if the sample was tilted away from the detector, the signal would fall to zero as the specimen holder cast a 'shadow' on the detector. Nevertheless, some early heroic attempts ${ }^{13,14}$ were made to use EDXS detectors with this asymmetric geometry to determine 3D composition from a very limited tilt series of EDXS data.

Over the past few years, however, there has been something of a revolution in EDXS detector design. Modern silicon drift detectors, with much higher solid angles of acceptance (ca. $0.9 \mathrm{sr}$ ) and with faster processing capabilities (100 k counts $\mathrm{s}^{-1}$ ) are now widely available for (S)TEM. These can be arranged with multiple detector chips positioned symmetrically about the optic axis, so that there are always chips 'facing' the sample no matter the tilt direction. This has led to a renaissance of EDXS tomography.

Figure 3 (a) shows a recent example of a nano-ring structure composed of Au and Ag. ${ }^{15}$ Here the two elements were mapped with nanometer resolution to determine their distribution from different starting alloys and how the two immiscible materials separated over time. Figure 3(b) shows another, more complex nanostructure, in which a $\mathrm{ZnO}$ shell covers a metal/carbon rich inner core. ${ }^{16}$ The complex arrangement of metallic features is revealed by the $3 \mathrm{D}$ elemental 
reconstruction. Moreover, in this case, quantification of the structure's composition was improved through absorption correction. Absorption of low energy X-rays is a major problem in any EDXS analysis and especially so for 3D EDXS, as the structures examined are likely to be thicker than would be the case for a thin film characterization. A number of methods have been explored in the past, ${ }^{17}$ improving upon the standard Cliff-Lorimer approach, including the zeta factor method, but here the absorption was corrected in an iterative fashion using tabulated mass-absorption coefficients, applying the correction voxel by voxel until a consistent set of composition values were obtained.

In modern instruments, it is possible to collect simultaneously the EDXS and EELS signals. Figure 3(c) exemplifies such combined data collected from a needle shaped sample of an Al-Si alloy (Al-5 wt\% Si with 50 ppm Na and 6100 ppm $\mathrm{Yb}$ ), showing the distribution of the metallic constituents. ${ }^{18}$ The great advantage of simultaneous acquisition is that the EELS signal is especially sensitive to light elements, the EDX to heavier elements, so the combination can provide a more complete $3 \mathrm{D}$ chemical picture.

\section{Crystallographic Electron Tomography}

Electron diffraction may also be regarded as a form of analytical electron microscopy. Through improvements in goniometer design, microscope control and detectors, and increased computational speed for analysis, this form of analytical microscopy has also had a renaissance in recent years, and is now being extended to AET.

In one variant of 'diffraction tomography', fixed-beam diffraction patterns are recorded as a function of specimen tilt, yielding a greatly increased reciprocal space data set that can be used for 3D structure solution and crystal analysis. ${ }^{19}$ Alternatively, by recording a tilt series of crystallographically-sensitive images, a full 3D picture of the local crystallography may be obtained. The first example of 
this using a TEM, shown in Figure 4 (a), provided a 3D reconstruction of the grains of a polycrystalline $\mathrm{Al}$ specimen. ${ }^{20}$ To achieve the reconstruction, ca. $100 \mathrm{k}$ dark-field (DF) images were recorded using a method analogous to one that has been successful in X-ray diffraction contrast tomography (DCT), varying sample tilt and the scattering angle for each DF image.

A somewhat different method involves coupling electron diffraction with scanning. Here, 2D maps of local crystallographic information can be obtained that are akin to those achieved with the widely adopted scanned electron backscatter diffraction (EBSD) technique. Acquisition involves recording a diffraction pattern at every pixel in a 2D scan, yielding a 4D data set, and the approach can be extended to tomography by repeating this process at different tilts. ${ }^{21}$ Such tilt series data can be extremely information-rich, and allow versatile 3D crystallographic analysis. The diffraction patterns can be analyzed computationally post facto, and 'virtual' DF or 'component' images may be formed (the latter using MSA methods; see below), which can then be used to reconstruct, in 3D, both real and reciprocal spaces, as illustrated in Figure 4 (b). Moreover, by interrogating sub-volumes to retrieve local 3D crystallography, it is possible to determine, for example, the orientation relationships between grains or phases and across interfaces.

\section{Advanced AET}

The 'multi-dimensional' nature of AET tends to lead to large data sets; it is quite typical to record ca. $100 \mathrm{k}$ spectra or diffraction patterns in one data set. As such, there is a need to optimize data analysis and to use data reduction techniques in order to extract the salient information about the sample. Multivariate statistical analysis (MSA) methods lend themselves to such 'big data' challenges, and have proven to be successful for AET. Figure 5 (a) shows a composite reconstruction of five surface plasmon modes determined by STEM- EELS tomography. ${ }^{22}$ In order to achieve such a reconstruction, the data was analyzed using non-negative 
matrix factorization (NMF), a form of MSA, so that each of the ca. $100 \mathrm{k}$ raw spectra could be described using a linear combination of just five surface plasmon spectral components. Reducing the spectral information to a series of key component spectra allows a much more interpretable evaluation of the enormous data sets. Closely related from a materials perspective, but differing in methodology, a complementary AET technique, cathodoluminescence tomography, was used recently to generate 3D surface plasmon maps from Ag spheres, see Figure 5(b). ${ }^{23}$

Although AET data sets are large, they are still incomplete from a tomographic perspective and the reconstructions will suffer artefacts. The electron dose and acquisition time required for analytical transmission electron microscopy is typically much larger than in standard imaging, which restricts the number of images that can be acquired in an AET tilt series - and hence the angular sampling from a tomographic reconstruction perspective. This has led to AET being used primarily to investigate materials that can withstand large electron doses without damage, but this situation may be improved if advanced reconstruction procedures are employed. ${ }^{22,24,25}$ In particular, novel reconstruction algorithms are under development that move away from the simple backprojection approach, to ones in which a model reconstruction is refined via a bestfit procedure using both the data and additional constraints based on prior knowledge about the object being reconstructed. The prior knowledge may be physical, for example imposing physical laws or zero background values, or it may be image-related, such as knowledge of the likely grey scale 'texture'. Such prior knowledge is extremely valuable in improving the fidelity of the tomogram and reducing the number of images required for a successful reconstruction, especially when implemented within the framework of so-called 'compressed sensing' methods. ${ }^{26}$ It is also possible to incorporate more complex beam-sample interactions into forward model-fitting calculations, an example of which is shown in Figure 5(c). ${ }^{24}$ As with Figure 5(a), a series of low-loss EELS spectrum images were recorded and MSA methods used to determine surface plasmon 
modes. However, here, the model-fitting approach, matching simulated and experimental EEL spectra, was necessary for refinement of the underlying charge density of the individual eigenmodes. This type of approach opens the way to determine many other physical properties in 3D that are not simple projections and thus not directly reconstructable using back-projection methods.

\section{Conclusions}

AET has led to new insights into the 3D structure, composition and physicochemical nature of materials. The development of novel AET techniques has gained considerable momentum in recent years. It is now possible to acquire vast 'multi-dimensional' spectrum-imaging and diffraction-imaging data sets that require analysis in an efficient and robust fashion. Machine learning and related techniques offer powerful ways to achieve this, and coupled with novel tomographic acquisition and reconstruction approaches provide 3D nanoscale information not available with any other technique.

\section{Acknowledgments}

The research leading to these results has received funding from the European Union Seventh Framework Programme under Grant Agreement 312483ESTEEM2 (Integrated Infrastructure Initiative-I3), as well as from the European Research Council under the European Union's Seventh Framework Programme (FP/2007-2013)/ERC grant agreement 291522-3DIMAGE. RKL acknowledges a Junior Research Fellowship at Clare College.

\section{Figure Captions}

Figure 1. Principle of analytical electron tomography, in which each voxel in 3D space contains additional signal dimensionality. This could be a specific energy from spectroscopy, or the full spectrum, or reciprocal space information. Upon interrogation, these signals may be used for spatial discrimination of specific elements, phases or other properties such as plasmonics. 
Figure 2. (a) EFTEM tomography elemental mapping of an iron-filled multiwalled carbon nanotube; red=iron, yellow=carbon. ${ }^{6}$ (b) STEM-EELS tomography chemical state mapping of silicon in a W-to-Si contact from a semiconductor device. ${ }^{9}$ (c) EFTEM tomography plasmon mapping, revealing the morphology of non-spherical silicon nanoparticles embedded in silicon oxide. (d) STEM-EELS tomography valence state mapping of a ceria nanoparticle. ${ }^{11}$

Figure 3. (a) 3D elemental maps of Ag-rich (left) and more Au-rich (right) bimetallic nano-rings, showing irregular Au surface segregation compared to uniform Ag surface segregation, respectively; obtained from STEM-EDXS tomography. ${ }^{15}$ (b) STEM-EDXS tomography reconstruction revealing 3D elemental distributions in an organic/inorganic core/multi-shell nano-wire. ${ }^{16}$ (c) 3D elemental maps of ytterbium (green), silicon (red) and aluminum (blue) from an Al-5 wt\%Si alloy (6100 ppm Yb), obtained from simultaneous STEM-EELS and STEM-EDXS tomography. ${ }^{18}$

Figure 4. (a) 3D grain-orientation map of a polycrystalline aluminum specimen (colors represent different crystal orientations), obtained from a hollow cone darkfield imaging approach to crystallographic tomography. ${ }^{20}$ (b) $3 \mathrm{D}$ crystallographic reconstruction of a Ni-based superalloy from scanning precession electron diffraction tomography. At each point in real space (left), reciprocal space information is available, as shown (right) for the lath-like $\eta$-phase rendered in green. $^{21}$

Figure 5. (a) 3D rendering of surface plasmon modes of a silver nano-cube, reconstructed using STEM-EELS tomography. ${ }^{22}$ (b) Cathodoluminescence spectroscopic tomography maps of a plasmonic gold-polystyrene nano-cresent. ${ }^{23}$ (c) Simulated plasmonic eigenmode (bottom) and corresponding STEM-EELS tomographic surface charge reconstruction (top) of a silver right bipyramid on a $\mathrm{MoO}_{3}$ dielectric substrate. ${ }^{24}$ 


\section{Author biographies}

\section{Rowan Leary}

Email: rk126@cam.ac.uk

Tel: +44(0)1223334597

Address: Department of Materials Science and Metallurgy, 27 Charles Babbage Road, University of Cambridge, Cambridge, CB3 OFS, UK.

Rowan Leary obtained his MEng/BEng in Materials Science and Engineering at the University of Leeds, and his $\mathrm{PhD}$ from the University of Cambridge. He is currently a Junior Research Fellow at Clare College and the Department of Materials Science and Metallurgy, University of Cambridge. His research involves the development of a variety of multi-dimensional electron microscopy techniques, and their application in the context of materials chemistry and catalysis in particular.

\section{Paul Midgley}

Email:pam33@cam.ac.uk

Tel: +44(0)1223334561

Address: Department of Materials Science and Metallurgy, 27 Charles Babbage Road, University of Cambridge, Cambridge, CB3 OFS, UK.

Paul Midgley is Professor of Materials Science and Director of the Wolfson Electron Microscopy Suite at the Department of Materials Science and Metallurgy, University of Cambridge. He is a Fellow of the Royal Society and a Professorial Fellow at Peterhouse. He has studied a wide variety of materials by electron microscopy and developed a number of novel electron microscopy techniques, in particular electron tomography and precession electron diffraction. 


\section{References}

1. R. Leary, P. A. Midgley, J. M. Thomas, Acc. Chem. Res. 45, 1782-1791 (2012).

2. P. A. Midgley, R. E. Dunin-Borkowski, Nat. Mater. 8, 271-280 (2009).

3. P. Ercius, O. Alaidi, M. J. Rames, G. Ren, Advanced Materials 27, 56385663 (2015).

4. O. Ersen, I. Florea, C. Hirlimann, C. Pham-Huu, Materials Today 18, 395408 (2015).

5. D. Wolf, A. Lubk, F. Röder, H. Lichte, Curr. Opin. Solid St. M. 17, 126134 (2013).

6. N. Y. Jin-Phillipp, C. T. Koch, P. A. van Aken, Ultramicroscopy 111, 1255-1261 (2011).

7. $\quad$ G. Mobus, B. J. Inkson, Appl. Phys. Lett. 79, 1369-1371 (2001).

8. M. Weyland, P. A. Midgley, Institute of Phyics Conference Series (EMAG 2001) 161, 239-242 (2001).

9. K. Jarausch, P. Thomas, D. N. Leonard, R. Twesten, C. R. Booth, Ultramicroscopy 109, 326-337 (2009).

10. A. Yurtsever, M. Weyland, D. A. Muller, Appl. Phys. Lett. 89, 151920 (2006).

11. B. Goris, S. Turner, S. Bals, G. Van Tendeloo, ACS Nano 8, 10878-10884 (2014).

12. R. F. Egerton, Electron Energy-Loss Spectroscopy in the Electron Microscope. (Springer, New York, 2011).

13. G. Möbus, R. C. Doole, B. J. Inkson, Ultramicroscopy 96, 433-451 (2003).

14. Z. Saghi, X. Xu, Y. Peng, B. Inkson, G. Mobus, Appl. Phys. Lett., 251906251901-251903 (2007).

15. T. J. A. Slater, A. Macedo, S. L. M. Schroeder, M. G. Burke, P. O'Brien, P. H. C. Camargo, S. J. Haigh, Nano Lett. 14, 1921-1926 (2014).

16. P. Burdet, Z. Saghi, A. N. Filippin, A. Borrás, P. A. Midgley, Ultramicroscopy 160, 118-129 (2016).

17. M. Watanabe, D. B. Williams, J. Microsc. 221, 89-109 (2006).

18. G. Haberfehlner, A. Orthacker, M. Albu, J. Li, G. Kothleitner, Nanoscale 6, 14563-14569 (2014).

19. U. Kolb, E. Mugnaioli, T. E. Gorelik, Cryst. Res. Technol. 46, 542-554 (2011).

20. H. H. Liu, S. Schmidt, H. F. Poulsen, A. Godfrey, Z. Q. Liu, J. A. Sharon, X. Huang, Science 332, 833-834 (2011).

21. A. S. Eggeman, R. Krakow, P. A. Midgley, Nat. Commun. 6, 7267 (2015).

22. O. Nicoletti, F. de la Pena, R. K. Leary, D. J. Holland, C. Ducati, P. A. Midgley, Nature 502, 80-84 (2013).

23. A. C. Atre, J. M. BrennyBenjamin, T. Coenen, A. García-Etxarri, A. Polman, J. A. Dionne, Nat. Nano. 10, 429-436 (2015). 
24. S. M. Collins, E. Ringe, M. Duchamp, Z. Saghi, R. E. Dunin-Borkowski, P. A. Midgley, ACS Photonics 2, 1628-1635 (2015).

25. Z. Saghi, M. Benning, R. Leary, M. Macias-Montero, A. Borras, P. A. Midgley, Adv. Struct. Chem. Imag. 1, 1-10 (2015).

26. R. Leary, Z. Saghi, P. A. Midgley, D. J. Holland, Ultramicroscopy 131, 70-91 (2013). 